\title{
Numerical Modeling of Functionally Graded Coatings
}

\author{
Maryam Heidari ${ }^{1,}$, Maria Kashtalyan ${ }^{1, b}$ \\ ${ }^{1}$ Centre for Micro- and Nanomechanics (CEMINACS), School of Engineering, \\ University of Aberdeen, Fraser Noble Building, Aberdeen AB24 3UE, UK \\ ar05mh0@abdn.ac.uk, ${ }^{\mathrm{a}} \mathrm{m} . \mathrm{kash}$ talyan@abdn.ac.uk
}

Keywords: functionally graded coatings, numerical modeling, finite element modelling

\begin{abstract}
Coatings play an important role in a variety of engineering applications protecting metallic or ceramic substrates against oxidation, heat penetration, wear and corrosion. One of the contributing factors to structural or functional failure of coatings is a mismatch of material properties between the coating and substrate at the coating/substrate interface. The concept of Functionally Graded Material (FGM) is actively explored in coating design for the purpose of eliminating this mismatch and improving coating performance and integrity. This paper presents analysis of the mechanical behavior of functionally graded coatings using commercial finite elements software ABAQUS in which user implemented graded finite elements have been employed. The model is used to carry out a comparative study of three-dimensional stress and displacement fields in the coated plates with homogeneous and functionally graded coatings.
\end{abstract}

\section{Introduction}

Functionally graded materials (FGMs) are heterogeneous composite materials with gradual variation of the constituents (e.g. metallic and ceramic) from one surface of the material to the other which results in continuously varying material properties [1]. FGMs have a number of advantages that make them attractive in potential applications, such as a potential reduction of in-plane and transverse through-the-thickness stresses, an improved residual stress distribution, enhanced thermal properties, higher fracture toughness, and reduced stress intensity factors [2].

FGMs represent a rapidly developing area of materials science and engineering with numerous practical applications. In coating design, the FGM concept can be used to eliminate the mismatch of material properties at the coating/ substrate interface, typical of conventional coatings, and thus increase coating's resistance to functional failure in the form of cracking and debonding.

From the modelling point of view, FGMs represent a challenge since continuous variation of material properties leads to significant analytical and numerical difficulties. A review of recent advances in the area of modelling and analysis of FGMs and structures is given in [2]. This paper presents a Finite Element model developed for investigation of the three-dimensional elastic deformation of functionally graded coatings under various loading conditions.

\section{Methodology}

The Finite Element Method used in the present paper employs elements with stiffness gradient in the thickness direction. Graded isoparametric elements are defined through the user material subroutine UMAT. The method of element formulation is discussed in detail elsewhere [3-5]. In the Finite Element Method, the displacement within an element can be written as shape functions,

$$
u(x)=\sum_{i=1}^{n} N_{i}(x) U_{i}
$$


where $N_{i}(x)$ is a matrix of shape functions corresponding to each of the $n$ nodes of the element and $U_{i}$ are nodal displacements corresponding to each of the $n$ nodes. For linear elastic behavior standard stress-strain relations can be used,

$$
\sigma(x)=C(x) \varepsilon(x)
$$

where $C(x)$ is the material property matrix. ABAQUS does not support graded elements therefore they should be defined in a user subroutine. The property matrix for the functionally graded material should be set to a spatially variable function. Exponential, power law and linear functions can be used to capture the variation of stiffness gradient in coating. Here, the exponential variation in shear modulus is used in order to allow comparison with available analytical solutions. The input file is consisting of the defined geometry, type of material properties, applied forces and boundary conditions. Implementing the graded features using the subroutine within the input file provides the mechanical constitutive behavior of the material in all calculation nodes.

\section{Results and discussion}

As an example, a simply supported coated plate with dimensions $a / h=b / h=10$ and the coating thickness $h^{(2)}=0.1 h$ is considered. The loading, $Q\left(x_{1}, x_{2}\right)$, is applied to the upper surface of coating, while the bottom surface of the plate remains free (Fig. 1). It is assumed that the loading can be expressed as a double Fourier series

$$
Q\left(x_{1}, x_{2}\right)=\sum_{m=1}^{\infty} \sum_{n=1}^{\infty} q_{m n} \sin \frac{\pi m x_{1}}{a} \sin \frac{\pi n x_{2}}{b}
$$
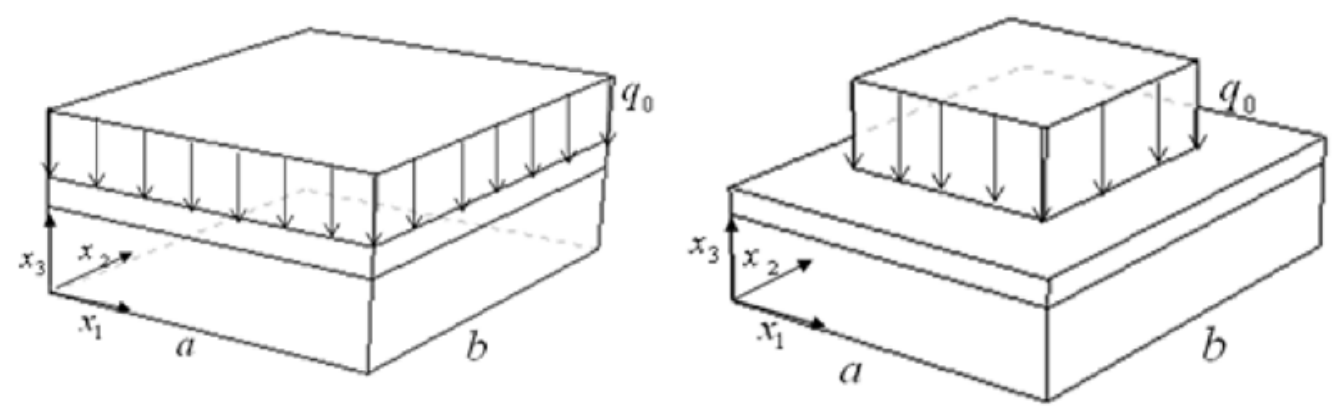

Figure 1. Coated plate subjected to UDL and patch loading relative to Cartesian coordinates.

Various loading schemes can be investigated using this representation. In particular, uniformly distributed loading (UDL) $Q\left(x_{1}, x_{2}\right)=q_{0}$ (Fig. 1) yields

$q_{m n}=\frac{16 q_{0}}{\pi^{2} m n}, \quad m=n=1,3,5, \ldots$

For patch loading (Fig. 1) $Q\left(x_{1}, x_{2}\right)=q_{0}$ applied at the centre of the plate $-c \leq x_{1} \leq c,-d \leq x_{2} \leq d$, we have 
$q_{m n}=\frac{16 q_{0}}{\pi^{2} m n} \sin \frac{\pi m x_{0}}{a} \sin \frac{\pi n y_{0}}{b} \sin \frac{\pi m c}{a} \sin \frac{\pi n d}{b}, \quad m=n=1,3,5, \ldots$

In the numerical calculations, the infinite series, Eq. (3), is replaced with the finite sum, with the number of terms in the sum determined from convergence studies. For UDL and patch loading, up to 29 terms in $m, n$ is required to achieve acceptable convergence.

The shear moduli of the substrate $(k=1)$ and the coating $(k=2)$ vary exponentially through the thickness according to

$$
G^{(k)}\left(x_{3}\right)=g^{(k)} \exp \left(\gamma^{(k)} \frac{x_{3}}{h}\right), \quad k=1,2
$$

where $\gamma^{(\mathrm{k})}$ are the inhomogeneity parameters, and $g^{(k)}=G^{(k)}(h)$. The Poisson ratios of the substrate and coating are equal to $v^{(1)}=v^{(2)}=0.3$.

The developed Finite Element model for graded material is validated through comparison with the three dimensional solution for isotropic graded plate [6]. Up to 40 nodes in the thickness direction are required to achieve good agreement with the analytical solution. Also, for one-term sinusoidal loading, the present Finite Element solution is found to be in an excellent agreement with the 3-D elasticity solution for coated plate [7]. Figures 2-4 show through-thickness variation of the normalised stresses $\bar{\sigma}_{i j}=\sigma_{i j} / q_{0}$ and normalised displacements $\bar{u}_{i}=\frac{G_{C}^{(2)} u_{i}}{q_{0} h}$ at the centre of two systems under UDL: one with the homogenous coating (HC) and the other with functionally graded coating (FGMC). For the out-of-plane normal stress $\bar{\sigma}_{33}$, through thickness variation is similar for both types of coating indicating the type of coating has no effect on this stress component. The transverse shear stress, $\bar{\sigma}_{13}$, in the FGMC system is close to parabolic profile, while in the HC system $\bar{\sigma}_{13}$ has a peak which is eliminated when homogeneous coating is replaced with graded coating. The type of coating has a strong effect on $\bar{\sigma}_{11}$ and $\bar{\sigma}_{12}$; it can be seen that in FGMC system there is no discontinuity across the coating/substrate interface (Fig. 3).

A

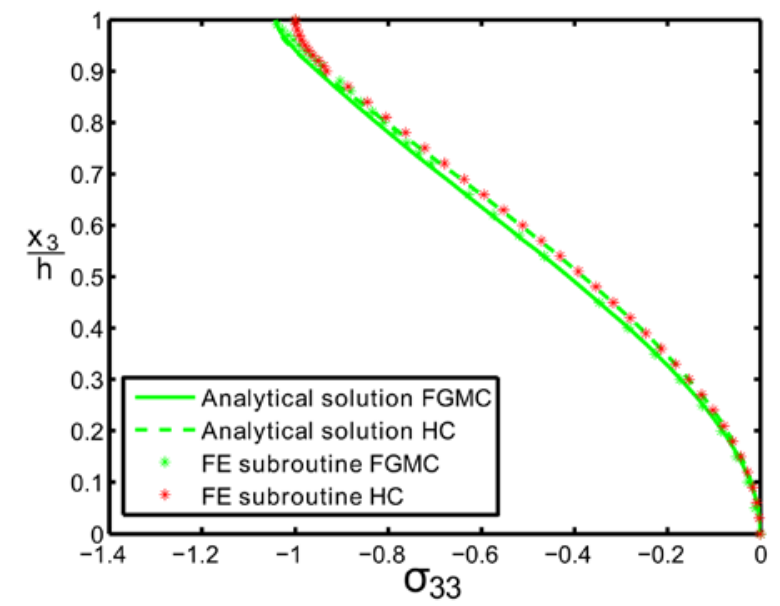

B

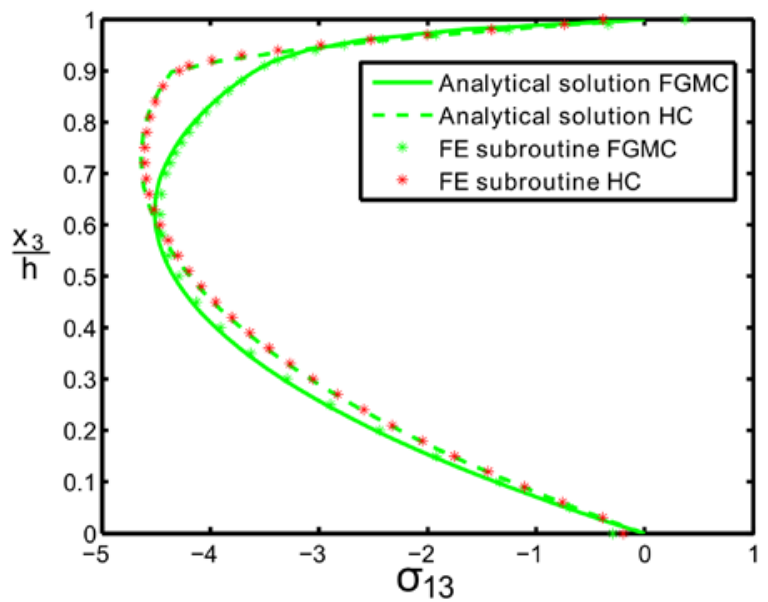

Figure 2 Through-thickness variation of (A) the normalised out-of-plane normal stress $\bar{\sigma}_{33}\left(0.5 a, 0.5 b, x_{3}\right)(\mathrm{B})$ the normalised transverse shear stress $\bar{\sigma}_{13}\left(0,0.5 b, x_{3}\right)$ under UDL as predicted by the Finite Element (FE) model and the analytical solution. 
A

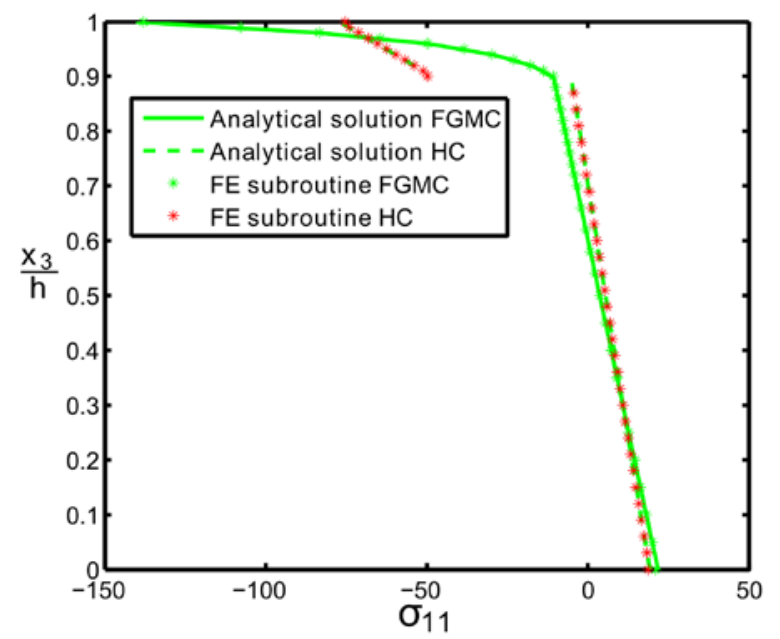

B

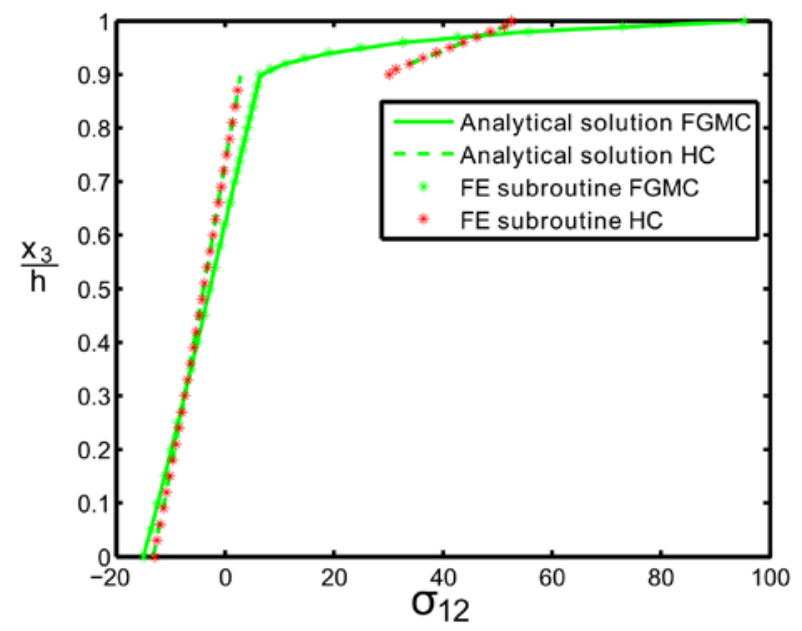

Figure 3 Through-thickness variation of (A) the normalised in-plane normal stress $\bar{\sigma}_{11}\left(0.5 a, 0.5 b, x_{3}\right)$ (B) the normalised in-plane shear stress $\bar{\sigma}_{12}\left(0,0, x_{3}\right)$ under UDL as predicted by the Finite Element (FE) model and the analytical solution.

A

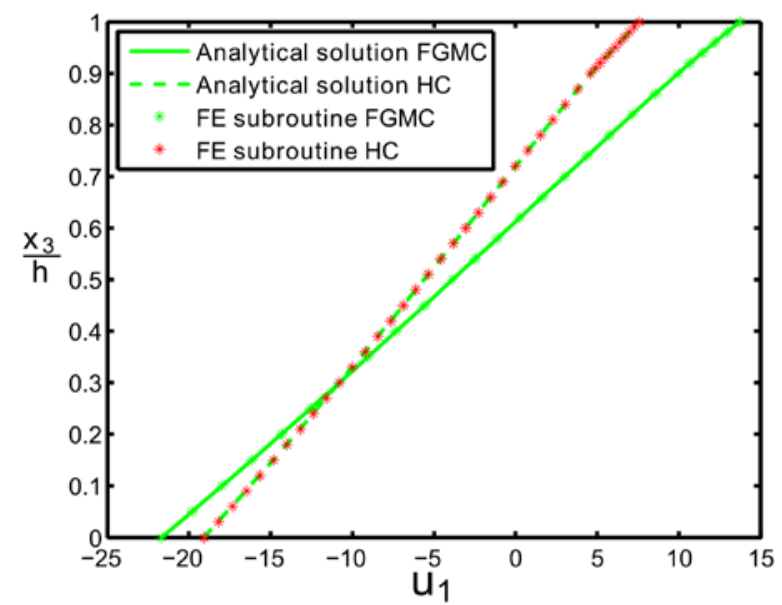

B

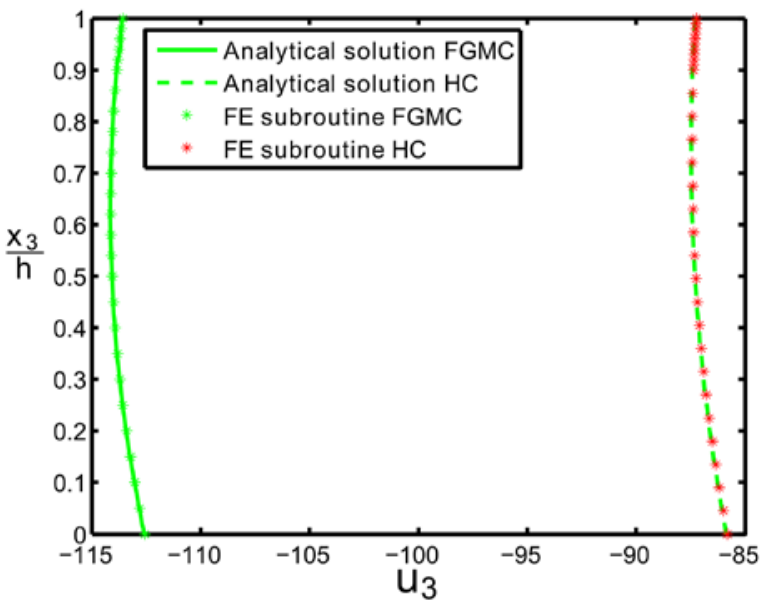

Figure 4 Through-thickness variation of (A) the normalised in-plane displacement $\bar{u}_{1}\left(0,0.5 b, x_{3}\right)$ (B) the normalised transverse displacement $\bar{u}_{3}\left(0.5 a, 0.5 b, x_{3}\right)$ under UDL as predicted by the Finite Element (FE) model and the analytical solution.

A

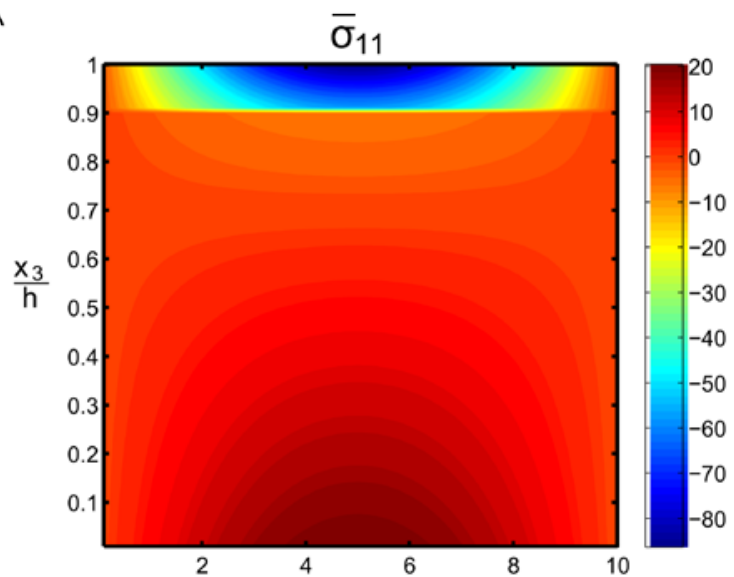

B

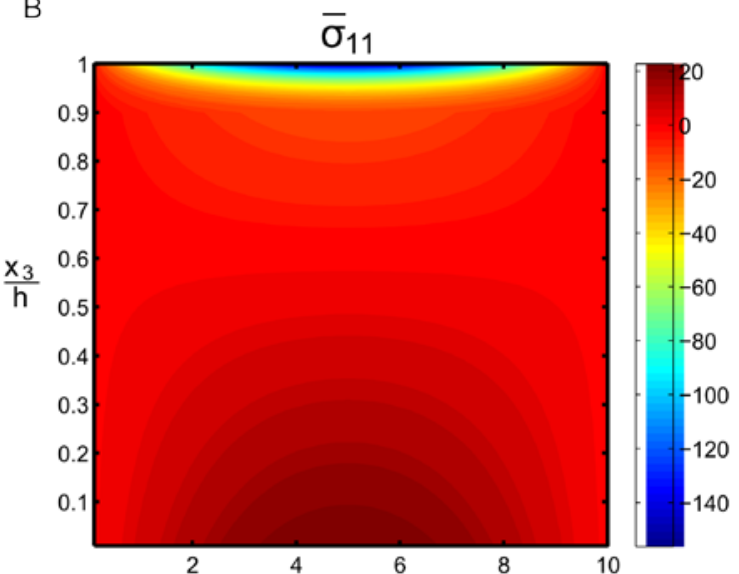

Figure 5 Through-thickness variation of the normalised in-plane normal stress $\bar{\sigma}_{11}\left(0.5 a, 0.5 b, x_{3}\right)$ for (A) HC and (B) FGMC systems under UDL as predicted by the FE model. 
A

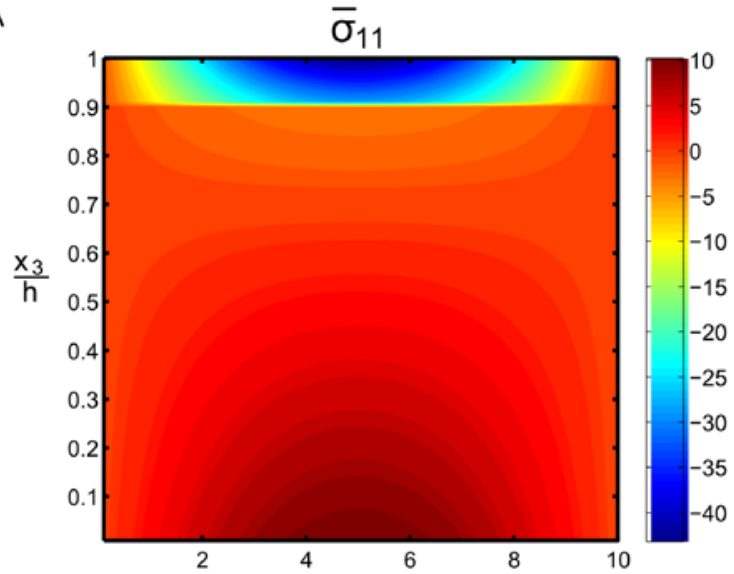

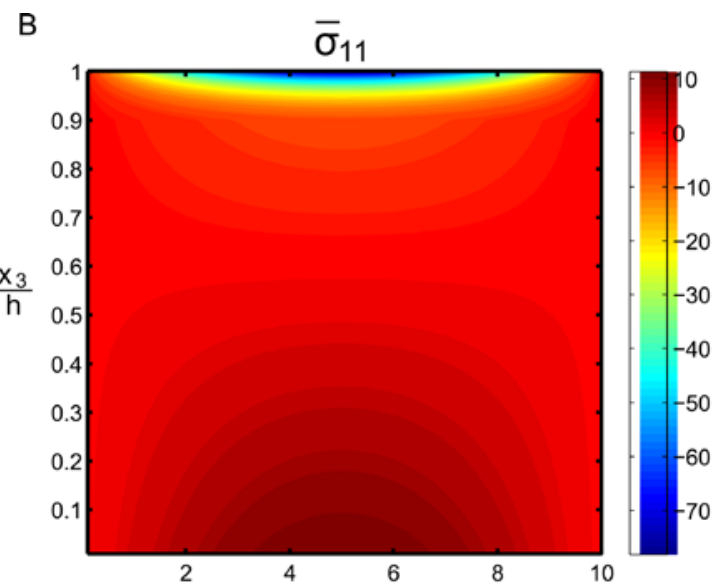

Figure 6 Through-thickness variation of the normalised in-plane normal stress $\bar{\sigma}_{11}\left(0.5 a, 0.5 b, x_{3}\right)$ for (A) HC and (B) FGMC systems under patch loading as predicted by the FE model.

Through-thickness variation of the in-plane displacement $\bar{u}_{1}$ in the FGMC and HC systems is linear and the differences are shown in Fig. 4A. The transverse displacement, $\bar{u}_{3}$, in the two systems is almost constant through the thickness which agrees well with the assumption of constant deflection made in some plate theories, however the type of coating has significant affect on $\bar{u}_{3}$, Fig.4B. Figures 5-6 show that the in-plane normal stress, $\bar{\sigma}_{11}$, changes from compressive at the top surface to the tensile at the interface in HC system while in the FGMC system it becomes compressive throughout the coating. The results are shown for the stiffness gradient $G_{c} / G_{s}=10$, where $G_{c}$ and $G_{s}$ are the shear moduli of the homogeneous substrate and homogeneous coating, respectively. In graded coating, the shear modulus varies from the $G_{s}$ value at the interface to the $G_{c}$ value at the top surface of the coating. In Fig. 6, the patch size is taken as $c=d=0.25$.

\section{Acknowledgements}

Financial support of this research by The Royal Society, The Royal Academy of Engineering, The Royal Society of Edinburgh and The Carnegie Trust for the Universities of Scotland is gratefully acknowledged.

\section{References}

1. S. Suresh, A. Mortensen. Fundamentals of Functionally Graded Materials. IOM Communications, London, 1998.

2. V Birman, L.W. Byrd. Modeling and analysis of functionally graded materials and structures. Appl Mech Review 2007; 60: 195-216.

3. W.G. Buttlar, G.H. Paulino, S.H. Song, Application of graded finite elements for asphalt pavements, J Eng Mech 2006; 132: 240-8.

4. J. Kim, G.H. Paulino, Isoparametric graded finite elements for nonhomogenous isotropic and orthotropic materials, J Appl Mech Trans ASME 2002; 69: 502-14.

5. M.H. Santare, J. Lambros, Use of graded finite elements to model the behavior of nonhomogenous materials, J Appl Mech 2000; 67: 819-22.

6. B. Woodward, M. Kashtalyan, Performance of functionally graded plates under localised transverse loading, Comp Struct 2012; 94: 2254-2264.

7. M. Kashtalyan, M. Menshykova, Three-dimensional elastic deformation of a functionally graded coating/substrate system, Int J Solids Struct 2007, 44: 5272-5288. 